\title{
ZRP versus AODV and DSR : A Comprehensive Study on ZRP Performance
}

\author{
Sree Ranga Raju \\ Department of Telecommunication \\ Bangalore Institute of Technology \\ Bangalore, India
}

\author{
Dr. Jitendranath Mungara \\ Ph.D \\ CMR Institute of Technology \\ Bangalore, India
}

\begin{abstract}
Mobile Ad Hoc Networks (MANETs) are networks of mobile computing nodes (e.g. portable computers, PDAs etc.) equipped with wireless interfaces and communicating with each other without relying on any infrastructure. In these networks each mobile node may act as a client, a server and a router. MANETs have emerged to fulfill the need for communication of mobile users in locations where deploying a network infrastructure is impossible, or too expensive, or simply is not available at that time. Characteristic scenarios for MANETs are disaster relief operations, battlefields and locations where infrastructure-based WLAN coverage (also called hotspots) is not provided and wireless WANs (e.g. GPRS/UMTS) are too expensive to use or too slow. Most of the research on MANETs has focused on issues dealing with the connectivity between mobile nodes in order to cope with the dynamism of such networks and the arising problems thereof. This dynamism is due to the mobility of nodes, the wireless channel's adverse and fast changing conditions and the energy limitations of mobile nodes, all of which lead to frequent disconnections and/or node failures. These research efforts have led to the creation of a sound technical basis for dealing with the aforementioned problems regarding node connectivity in MANETs (mainly through Zone Routing Protocol). However, solving the problems of connectivity alone is not sufficient for the adoption of MANETs. Since their basic role is to allow mobile users to exchange data and use each other's services, there is also a need for architectures, mechanisms and protocols to tackle route failures and minimize the control overhead.

One of well-known hybrid routing protocol for ad-hoc networks is Zone Routing Protocol (ZRP). However, in order to find its suitability, we needed to understand its behavior in various network sizes. So, in our survey we used QualNet 4.5.1 Network simulator to study the behavior of ZRP versus AODV and DSR. Their main characteristics have been presented and a thorough evaluation has been carried out for ZRP against DSR and AODV. Regretfully ZRP was not up to the task and it performed poorly throughout all the simulation sequences, hence putting itself out of competition. In particular, it demonstrated a really low packet delivery ratio when compared to DSR and AODV. From this survey, we could find that ZRP uses many control packets which are resulting in the increase of network load and decrease of network performance.
\end{abstract}

Keywords - Ad hoc, MANET, ZRP, DSR and AODV.

\section{INTRODUCTION}

Many routing algorithms for ad hoc wireless networks today promise rapid network convergence, multi-hop routing capabilities and soft real-time performance. With the use of IEEE 802.11 as the underlying Mac layer a totally distributed wireless infrastructure can self organize and form a multihop

wireless network. Some of the applications of ad-hoc networks can be in law enforcement, in emergency response in case of catastrophic events [1] as well as in various military applications. At the same time, a role for ad hoc networks exists also in construction sites, industry factories and public wireless networks in airports, stations, convention centers etc. The key factor that determines how efficiently a multihop wireless network reacts to topology changes and node mobility is the routing algorithm that provides routes for every node in the network. Several routing protocols have been proposed in the past both of proactive and reactive nature as well as and some that take a hybrid approach.

An analytical performance comparison of some of the most important algorithms is presented, like Dynamic Source Routing (DSR) [2],[3] and Adhoc Ondemand Distance Vector Routing Protocol (AODV) [4]. DSR is the main and most known protocol of the reactive family of protocols while AODV uses a unique approach in hop-by-hop routing, guiding every packet to its destination. We also evaluate Zone Routing Protocol (ZRP) [6] compared to the above protocols, which is a well known hybrid routing algorithm. The performance regarding end-to-end delay and number of packets successfully routed to their destination are examined. Finally the simulation results using QualNet are presented and evaluated in order to understand the strengths and weaknesses of ZRP.

\section{RELATED WORK}

There are several other efforts related to our work, with more recent work of Perkins, Royer, Samir R. Das and Manesh [7]. They evaluated DSR and AODV using NS-2 network simulator for 50 and 100 nodes in a rectangular space. The traffic and mobility models they used were the ones incorporated into NS-2. Their simulations didn't include ZRP, and also their work did not include study of impact of specific attributes of DSR or AODV in network performance. The mobility models were not different but instead they used a uniform distributed speed of nodes between 0-20 $\mathrm{m} / \mathrm{sec}$. This doesn't help to examine network performance in various mobility scenarios since the nodes move in a mean $10 \mathrm{~m} / \mathrm{sec}$ speed. Even more the physical medium model of IEEE 802.11 in NS-2 is not as sophisticated as that of QualNet model. Another related work has been presented by Broch, Maltz, Johnson, $\mathrm{Hu}$, and Jetcheva [8]. They evaluated four ad hoc routing protocols including AODV and DSR. They also used NS-2 to simulate 50node network models with mobility and traffic scenarios similar 
to the scenarios Perkins et al did. But their traffic loads are kept very low and they used packets of size 64 bytes only. Such small packet size is of course a disadvantage for DSR since when packet size is small, the source routing information inside every packet becomes more and more burdening for the network. On the other hand we used an exponentially distributed packet size of 512 by tes which makes the comparison fair between DSR, ZRP and ADOV. They concluded that DSR is a very good and superior protocol network in low traffic situations. The above results are extended by our work since we test DSR, as well as AODV and ZRP to all kind of conditions regarding node mobility and network loads. Besides comparison of ad hoc network several other papers have dealt with ZRP especially and tried to pinpoint the perfect zone radius value. Hass and Pearlman have done extensive research in ZRP and they have concluded that no fixed value of ZRP's zone radius attribute exists, but every time it is dependent on the networks conditions [9]. Even more they have shown that the use of further query control mechanism on ZRP enhances its performance in every network state [9]. The above work is also extended by trying to find the network conditions that make necessary the use of a higher or lower zone radius as well as IARP's Update Interval attribute, for ZRP to perform efficiently.

Last but not least there hasn't been any related work and presentation of simulation results, regarding how ZRP performs under varying properties like node density, area size, pathloss model, mobility, energy model, battery model, shadowing model and fading model.

\section{PROTOCOLS PRESENTATION}

We shall make a small presentation of the three protocols we evaluate in this paper. A more thorough presentation of ZRP shall be made, since it is mainly the impact of its attributes to its performance that we want to focus on this paper. The two other protocols have been simulated using the standard values, introduced in the corresponding drafts.

\subsection{Dynamic Source Routing (DSR)}

The Dynamic Source Routing protocol is composed of two main mechanisms to allow the discovery and maintenance of source routes in the ad hoc networks. Route Discovery: is the mechanism by which a source node wishing to send a packet to a destination node, obtains a source route to the destination. Route Discovery is used only when the source node attempts to send a packet to a destination and does not already know a route to that destination. Route Maintenance: is the mechanism by which a node wishing to send a packet to a destination is able to detect, while using a source route to the destination, if the network topology has changed. If this is the case then it must no longer use this route to the destination because a link along the route broken. Route Maintenance for this route is used only when the source node is actually sending packets to the destination. A routing entry in DSR contains all the intermediate nodes of the route rather than just the next hop information maintained in DSDV and AODV. A source puts the entire routing path in the data packet, and the packet is sent through the intermediate nodes specified in the path If the source does not have a routing path to the destination, then it performs a route discovery by flooding the network with a route request (RREQ) packet. Any node that has a path to the destination in question can reply to the RREQ packet by sending a route reply (RREP) packet. The reply is sent using the route recorded in the RREQ packet.
To limit the need for route discovery, DSR allows nodes to operate their network interfaces in promiscuous mode and snoop all (including data) packets sent by their neighbors. Since complete paths are indicated in data packets, snooping can be very helpful in keeping the paths in the route cache updated. To further reduce the cost of route discovery, the RREQs are initially broadcasted to neighbors only (zero-ring search), and then to the entire network if no reply is received. Another optimization feasible with DSR is the gratuitous route replies; when a node overhears a packet containing its address in the unused portion of the path in the packet header, it sends the shorter path information to the source of the packet (Automatic Route Shortening). Another important optimization includes the technique to prevent "Route reply Storms": because many route replies may be initiated simultaneously a delay time proportional to the hop's-distance can be used in order to give higher priority to near nodes. In addition a method called "Packet Salvaging" is often used in DSR. When an intermediate node forwarding a packet detects through Route Maintenance that the next hop along the route for that packet is broken, if the node has another route to the packets 's destination it uses it to send the packet rather than discard it. An interesting and a bit different approach of DSR is the DSR_flows described in [13]. To summarize we provide the basic characteristics of the Dynamic Source Routing (DSR):

- Uses source routing

- Provides loop-free routes

- Supports unidirectional links and asymmetric routes

- With the optimizations available it is a good choice for an ad hoc network

\subsection{Zone Routing Protocol (ZRP)}

Zone Routing Protocol or ZRP was the first hy brid routing protocol with both a proactive and a reactive routing component. ZRP was first introduced by Haas in 1997. ZRP is proposed to reduce the control overhead of proactive routing protocols and decrease the latency caused by routing discover in reactive routing protocols. ZRP defines a zone around each node consisting of its kneighborhood (e. g. k=3). In ZRP, the distance and a node , all nodes within -hop distance from node belongs to the routing zone of node . ZRP is formed by two sub-protocols, a proactive routing protocol: Intra-zone Routing Protocol (IARP), is used inside routing zones and a reactive routing protocol: Inter-zone Routing Protocol (IERP), is used between routing zones, respectively. A route to a destination within the local zone can be established from the proactively cached routing table of the source by IARP, therefore, if the source and destination is in the same zone, the packet can be delivered immediately. Most of the existing proactive routing algorithms can be used as the IARP for ZRP.

For routes beyond the local zone, route discovery happens reactively. The source node sends a route requests to its border nodes, containing its own address, the destination address and a unique sequence number. Border nodes are nodes which are exactly the maximum number of hops to the defined local zone away from the source. The border nodes check their local zone for the destination. If the requested node is not a member of this local zone, the node adds its own address to the route request packet and forwards the packet to its border nodes. If the destination is a member of the local zone of the node, it sends a route reply on the reverse path back to the source. The source node uses the path 
saved in the route reply packet to send data packets to the destination.

\subsection{Adhoc On demand Distance Vector Routing protocol (AODV)}

The AODV Routing protocol uses an on-demand approach for finding routes, that is, a route is established only when it is required by a source node for transmitting data packets. It employs destination sequence numbers to identify the most recent path. The major difference between AODV and Dynamic Source Routing(DSR) stems out from the fact that DSR uses source routing in which a data packet carries the complete path to be traversed. However, in AODV, the source node and the intermediate nodes store the next-hop information corresponding to each flow for data packet transmission. In an on-demand routing protocol, the source node floods the RouteRequest packet in the network when a route is not available for the desired destination. It may obtain multiple routes to different destinations from a single RouteRequest. The major difference between AODV and other on-demand routing protocols is that it uses a destination sequence number (DestSeqNum) to determine an up-to-date path to the destination. A node updates its path information only if the DestSeqNum of the current packet received is greater than the last DestSeqNum stored at the node.

A RouteRequest carries the source identifier (SrcID), the destination identifier (DestID), the source sequence number (SrcSeqNum), the destination sequence number (DesSeqNum), the broadcast identifier (BcastID), and the time to live (TTL) field. DestSeqNum indicates the freshness of the route that is accepted by the source. When an intermediate node receives a RouteRequest, it either forwards it or prepares a Route Reply if it has a valid route to the destination. The validity of a route at the intermediate node is determined by comparing the sequence number at the intermediate node with the destination sequence number in the RouteRequest packet. If a RouteRequest is received multiple times, which is indicated by the BcastID-SrcID pair, the duplicate copies are discarded. All intermediate nodes having valid routes to the destination, or the destination node itself, are allowed to send Route Reply packets to the source. Every intermediate node, while forwarding a RouteRequest, enters the previous node address and its BcastID. A timer is used to delete this entry in case a Route Reply is not received before the timer expires. This helps in storing an active path at the intermediate node as AODV does not employ source routing of data packets. When a node receives a Route Reply packet, information about the previous node from which the packet was received is also stored in order to forward the data packet to this next node as the next hop toward the destination.

\section{SIMULATION RESULTS}

In this section the methodology is presented .This methodology is used in order to isolate the impact on network performance.

\subsection{Simulation platform, models and attributes}

In our survey we have used the QualNet 4.5 simulator, including the wireless module to enable mobility of the wireless nodes and support more accurate wireless models for propagation, path loss, multipath fading and reception on wireless networks.the simulations were carried out under two phases, for network sizes of $10,25,50,100$ and 200 respectively. The area considered for the above network sizes are 500X500, (for network sizes of 10 and 25 nodes), 1000X1000 (for network sizes of 50 and 100 nodes respectively) and $3000 \times 3000$ (for network size of 200 nodes), for all the above specified network sizes the node placement strategy selected was "random node placement" under seed-1 so that the scenarios simulated represent a real life network. We had specified restricted mobility for selected nodes with speed limits ranging from $1 \mathrm{mps}$ to $8 \mathrm{mps}$. In this case either source or destination is mobile at any given time in simulation.

\subsubsection{Phase-1}

Phase-1 of the simulation was configured with the following parameters:

1. Routing Protocols: AODV, DSR and ZRP

2. Fading Model: Rayleigh

3. Shadowing Model: Constant

4. Pathloss Model: 2Ray

5. Energy Model: Simple Linear

6. Battery Model: Simple linear

7. Mobility: Restricted Mobility (for selected nodes)

8. Mobility Speeds: 1 to 8 meters per sec

\subsubsection{Phase-I}

Phase- 2 of the simulation was configured with the following parameters:

1. Routing Protocols: AODV, DSR and ZRP

2. Fading Model: Ricean

3. Shadowing Model: Long Normal

4. Pathloss Model: Freespace

5. Energy Model: MICAZ

6. Battery Model: Precise Service Life Estimator

7. Mobility: Restricted Mobility (for selected nodes)

8. Mobility Speeds: 1 to 8 meters per sec

The image of the network as it appears in QualNet 4.5 is presented in Figure-1. 


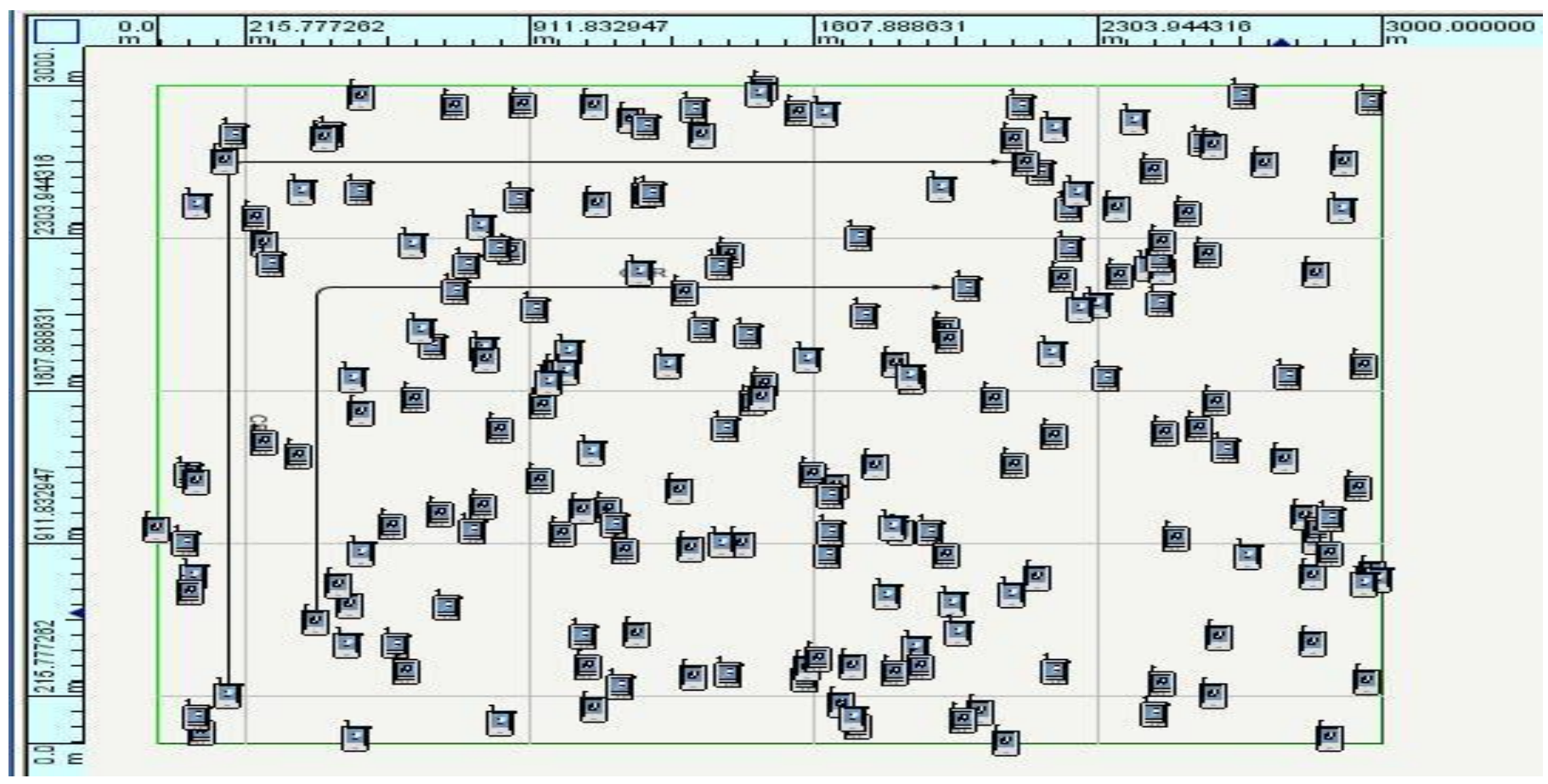

Figure-1: Topol ogy of the Simulated Network

The physical medium used is the well known 802.11 DSSS PHY with a data rate of $2 \mathrm{mbps}$. The M AC protocol used is also the 802.11 MAC protocol, configured in a MANET mode. More precisely we use only Distributed Coordination Function (DCF) of the protocol and not the Point Coordination Function (PCF), so there is no point coordinator and any centralized routing. It has been mentioned in many papers that IEEE 802.11 MAC protocol doesn't perform well in multihop topologies as well as in TCP connections [11], but since it is perhaps the most known and common to have been tested and implemented in hardware we choose to use it. In this paper we focus on the network layer protocol (routing sub-layer) and examine the performance of three of the most known routing protocols: DSR AODV and ZRP.

\subsection{Simulation sequences}

The graphs listed below from 1 thru 6 show the statistics like

1. Total packets received (out of 99)

2. Throughput (in bits per second)

3. Average end-to-end delay (in seconds)

4. Total by tes received (out of 50688)

5. Average Jitter (in seconds)

6. first packet received at (in seconds)

All the statistics were collected under Phase-1 (specified under section 4.1.1) over different network sizes of 10, 25, 50, 100 and 200 nodes respectively.

\section{packets received}

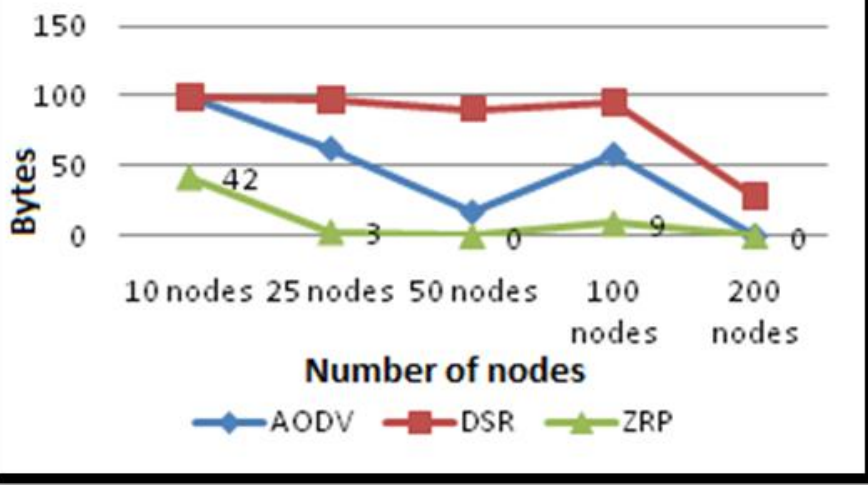

Table-1: shows the total packets received for multiple runs with different node density. 


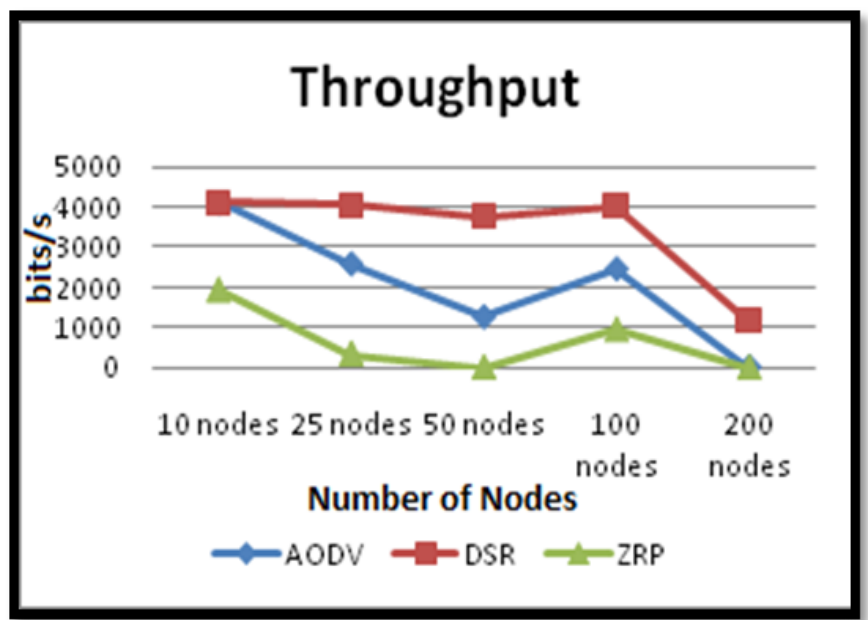

Table-2: Throughput in bits per second obtained for different network sizes.

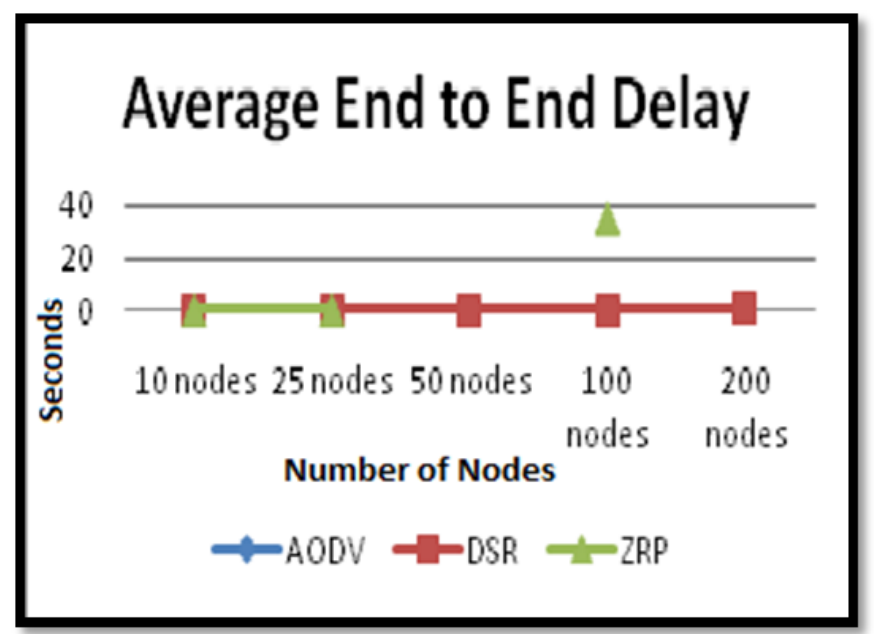

Table-3: Average End-toEnd delay displayed in seconds for different runs on different network sizes

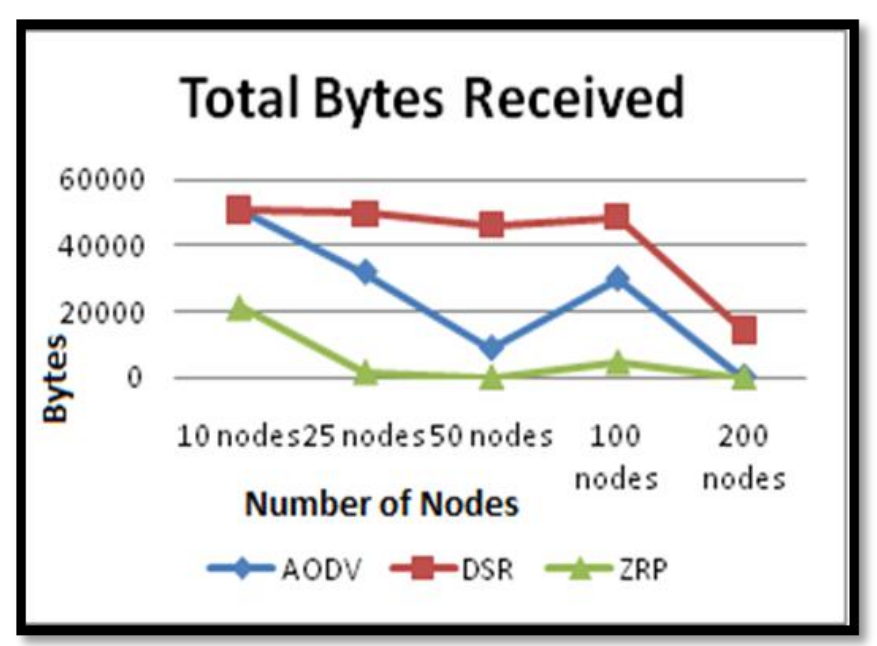

Table-4: Total Bytes Received out of total 50688 Bytes on different network sizes

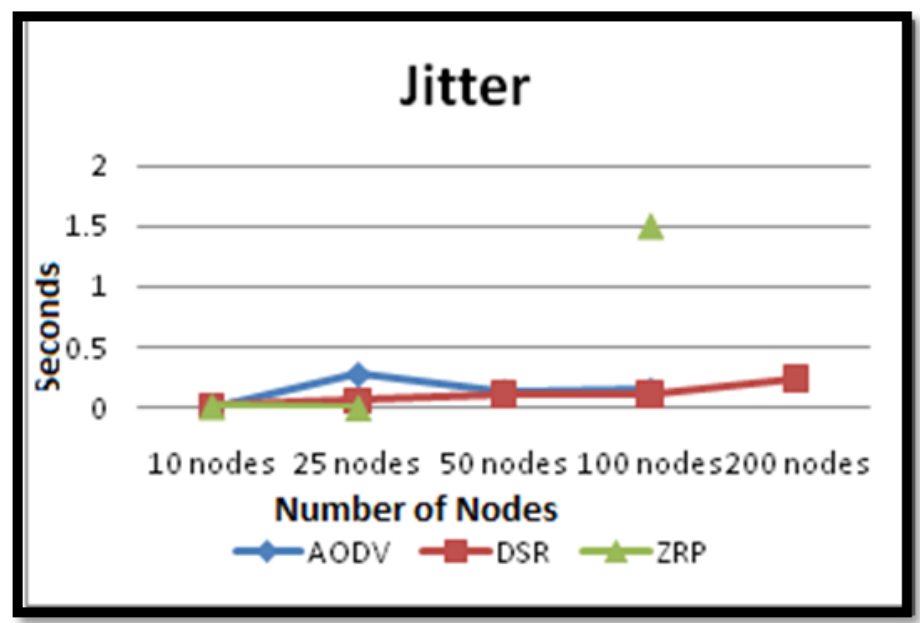

Table-5: The average jitter shown in seconds for different network sizes.

\section{First Packet Received at}

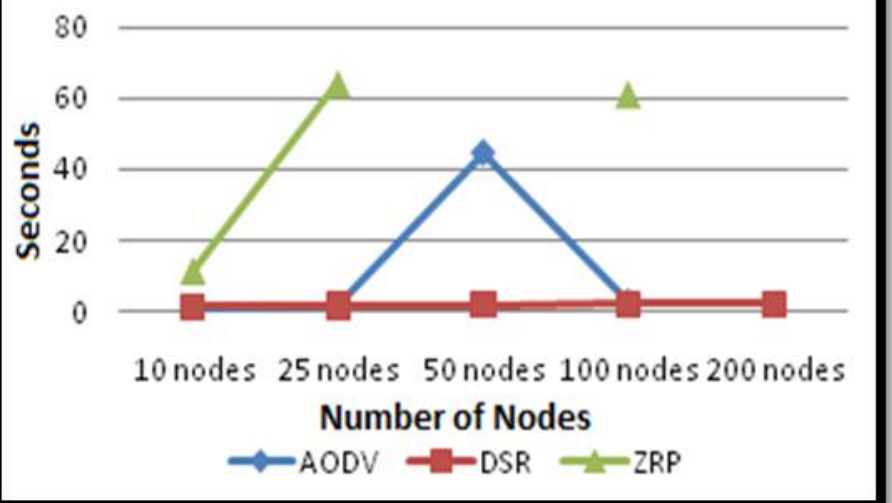

Table-6: shows when was the first packet received in seconds

\section{CONCLUSIONS}

Three important ad hoc routing protocols have been presented and evaluated through the well known QualNet Simulator. Their main characteristics have been presented and a thorough evaluation has been carried out for ZRP against DSR and AODV. Regretfully ZRP was not up to the task and it performed poorly throughout all the simulation sequences, hence putting itself out of competition. In particular it demonstrated a really low packet delivery ratio when compared to DSR and AODV. DSR on the other hand performed admirably and it would be the clear winner if not for its bad behavior in high traffic cases. AODV performed well in most of the network sizes (better than ZRP).

The results also throw a challenge and an excellent opportunity to look deeper into ZRP protocol and work a better algorithm that will improve the performance of ZRP.

Our future work shall propose a new algorithm to reduce the network load by limiting the number of control packets when the protocol searches for a new route. 
Routing protocols for mobile ad-hoc networks have to face the challenge of frequently changing topology, low transmission power and asymmetric links. Both proactive and reactive routing protocols prove to be inefficient under these circumstances. We shall propose a new algorithm which assumes that all routes in the routing table are active and usable, unless a broken link has been reported or discovered. There is no need for periodic updates of the routing table and control traffic is thus reduced. The new algorithm shall provide loop freedom, alternative paths in the case of route failure and disjoint paths. Within the zone, routes are immediately available, but for destinations outside the zone, the proposed algorithm shall use a route discovery mechanism to add routes to the table. In the case of link failure, the proposed algorithm shall use a unique field to identify routes affected by the failure.

\section{REFERENCES}

[1] "LOCCATEC: Low Catastrophic Event Capturing," European Research Project IST-2000-29401, www.loccatec.org

[2] D. Johnson, D. Maltz and Yih-Chun Hu. "The Dynamic Source Routing Protocol for Mobile Ad Hoc Networks," http://www.ietf.org/internet-drafts/draftietfmanet-DSR-09.txt, IETF Internet draft, Apr. 2003. Work in progress.

[3] David B. Johnson and David A. Maltz. "Dynamic source routing for ad hoc wireless networks". In Mobile Computing, edited by Tomasz Imielinski and Hank Korth, chapter 5, pages 153-181. Kluwer Academic Publishers, 1996.

[4] Vincent D. Park and M. Scott Corson. "Adhoc Ondemand Distance Vector (AODV) version 4: Functional specification". Internet-Draft,draft-ietfmanet- AODV-spec-04.txt, July 2001. Work in progress

[5] Vincent D. Park and M. Scott Corson. "A performance comparision of TORA and Ideal Link State routing." In Proceedings of IEEE Symposiumon Computers and communication '98, June 1998.

[6] Haas ZJ. "A new routing protocol for the reconfigurable wireless network. "In Proceedings of the 1997 IEEE 6th International Conference on Universal Personal Communications, ICUPC '97, San Diego, CA, October 1997; pp. 562-566.

[7] C. Perkins, E.M. Royer, S.R. Das, and M.K. Marina, "Performance Comparison of Two On-demand Routing Protocols for Ad Hoc Networks," IEEE Personal Communications, pp. 16-28, Feb. 2001
[8] J. Broch et al., "A Performance Comparison of Multihop Wireless Ad Hoc Network Routing Protocols," Proc. IEEE/ACM MOBICOM '98, Oct. 1998, pp. 85-97.

[9] Pearlman MR, Haas ZJ. "Determining the optimal configuration for the zone routing protocol." IEEE Journal on Selected Areas in Communications 1999; 17: 1395-1414.

[10] Z. J. Haas and M. R. Pearlman. "The performance of query controls chemes for the zone routing protocol." In Proceedings of the ACM SIGCOMM '98, pages 167-177. ACM Press, 1998.

[11] A. Ahuja et al., "Performance of TCP over different routing protocols in mobile ad-hoc networks,"Proceedings of IEEE Vehicular Technology Conference (VTC 2000), Tokyo, Japan, May 2000.

[12] A. Willig, M. Kubisch, C. Hoene, and A. Wolisz, "Measurements of a Wireless Link in an Industrial Environment using an IEEE 802.11-Compliant Physical Layer", IEEE Transactions on Industrial Electronics, vol. 43, no. 6, pp. 1265 1282, December 2002

[13] Yih-Chun Hu,D.B. Johnson, D. A. Maltz. "Flow State in the Dy namic Source Routing Protocol for Mobile Ad Hoc Networks “. IETF Internet Draft, Feb. 2001, Work in progress

\section{AUTHORS}

Dr. Jithendranath Mungara Ph.D, He has obtained M.Tech and Ph.D (Andhra University) and currently he is working as Dean Research and P.G Studies at CMRIT, Bangalore. He has served as Principal at BTLIT, Bangalore and Professor of Computer Science Department. He has teaching experience for more than a decade.

He has served as Engineer in BOEING U.S.A and software industries U.S.A

His areas of interest include MANETs, Sensor Networks and Cloud Computing.

Mr. Sree Ranga Raju has obtained B.E (B an galore University) in 1985 and M.Tech (Mysore University) in 1991 and currently he is a Research scholar in VMU, Salem Tamil Nadu, India.

He has teaching experience more than two and half decades. He has organised national conferences and workshop.

His areas of Research interest include Routing Protocols for Adhoc mobile networks, wireless Sensor Networks and Telecommunication Network Management. 